\title{
«Fragen Sie nicht, was die FMH für Sie tun kann - fragen Sie, was Sie für die FMH tun können»
}

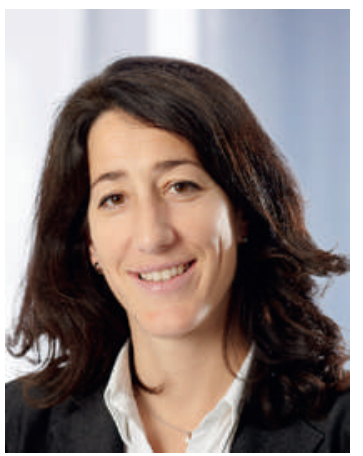

Mit diesen Worten, die an die berühmte Antrittsrede von John Fitzgerald Kennedy vom 20. Januar 1961 erinnern, wurden die Präsidentinnen und Präsidenten, Vorstandsmitglieder und Generalsekretäre zur Einführungsveranstaltung am 29. Oktober 2014 eingeladen. Bei diesem Treffen sollten die Teilnehmenden unter anderem nützliche Instrumente und Informationen für ihre neue Amtszeit erhalten. Neben den Strukturen der FMH, der Strategie 2013-2016 des Zentralvorstandes und den Aktivitäten seiner Departemente wurden die Tätigkeiten des Generalsekretariats vorgestellt, die im Dienst sämtlicher Mitglieder, der kantonalen Ärztegesellschaften sowie der Fachgesellschaften und Dachverbände stehen. Getreu dem Grundsatz «Tue Gutes und rede darüber» hat diese Veranstaltung die breite Palette an Aktivitäten im Jahr 2014 ergänzt, mit denen die FMH und ihre Dienstleistungen an die Mitglieder bekannter gemacht werden sollen. In diesem Sinn sieht die Strategie 2013-2016 vor, zum einen das Dienstleistungsangebot zu erfassen, zu analysieren und weiterzuentwickeln, zum anderen die Zusammenarbeit mit FMH Services zu verstärken sowie zum Schluss das Dienstleistungsangebot bekanntzumachen und zu fördern.

\section{Mehr als 100 direkte Dienstleistungen zugunsten aller Mitglieder.}

Eines der von uns im Laufe des Jahres 2014 verfolgten und auch erreichten Ziele bestand darin, jede einzelne Leistung zu analysieren, welche das Generalsekretariat für die Mitglieder und ihre Ärztegesellschaften erbringt, sei sie direkter oder indirekter Natur. Wir haben ihre Relevanz und Priorität überprüft und ihre Kosten verifiziert. Das Ergebnis ist eine Liste mit annähernd 100 Leistungen, von denen die Mitglieder direkt profitieren können. Diese gesamte Tätigkeit wird in einem Leistungskatalog zusammengefasst, der im nächsten Jahr erstellt und an die Mitglieder verteilt wird. Dieser Katalog wird auch die Leistungen von Partnern wie zum Beispiel FMH Services enthalten, dem bevorzugten Dienstleister der FMH.

Um die Vorteile einer Mitgliedschaft bei der FMH voll ausschöpfen zu können, muss man sie in erster Linie gut kennen. Deshalb unternehmen wir in dieser Amtszeit grosse Anstrengungen, um die FMH sichtbarer, zugänglicher und vorteilhafter für ihre Mitglieder zu gestalten. Neben der Analyse und der klaren Kommunikation unserer Leistungen sowie der Organisation von Veranstaltungen befassen wir uns mit der Stärkung der Marke der FMH und ihrer Präsenz vor Ort. So konnten Sie zum Beispiel den neuen Stand der FMH auf den verschiedenen Kongressen besuchen, an denen wir teilgenommen haben (siehe S. 1963 in der vorliegenden Ausgabe). Aber die Tätigkeit der FMH ist bei weitem nicht bloss eine Sache ihrer Organe oder ihres Führungsstabs. Unser Berufsverband setzt sich aus zahlreichen Kommissionen, Ausschüssen und Arbeitsgruppen zusammen, die zentrale Fragen zugunsten der Interessen der Ärzteschaft bearbeiten. Derzeit sind rund 169 Kommissionen aktiv, die aus Organen, Projekten oder Departemente der FMH hervorgehen. Der Einsatz der Mitglieder in diesen verschiedenen Kommissionen entspricht in etwa 200 Sitzungen pro Jahr mit ungefähr 800 Teilnehmenden.

Die FMH ist nichts anderes

als die Summe der ihr angegliederten medizinischen Gesellschaften und ihrer Mitglieder.

Und genau hier wird der Sinn der Einladung an die Mitglieder der FMH, an die Delegierten der ihr angegliederten medizinischen Gesellschaften und an die Vertreterinnen und Vertreter ihrer Organe deutlich: Fragen Sie nicht, was die FMH für Sie tun kann (wir werden Ihnen ohnehin ständig davon berichten!) - fragen Sie, was Sie für die FMH tun können. Denn die FMH ist nichts anderes als die Summe ihrer Mitglieder, und das Engagement jeder und jedes Einzelnen bringt die Sache der Ärzteschaft in einem nationalen gesundheitspolitischen Umfeld voran, welches der Stimme der Ärztinnen und Ärzte wenig Gehör schenkt. Die Veranstaltung vom 29. Oktober, über die Sie in dieser Ausgabe der SÄZ einen Bericht lesen können (siehe S. 1959), war ein voller Erfolg. Sie zählte 71 Teilnehmende und wurde von 89\% der Personen, die den Feedback-Fragebogen zurückschickten, als gut bis sehr gut bewertet. 97\% beurteilten den Inhalt als nützlich bis sehr nützlich. 82\% der Teilnehmenden empfanden die Dauer der Veranstaltung als angemessen, und 68\% empfehlen, sie jährlich zu wiederholen.

Ich möchte allen Mitarbeitenden des Generalsekretariats meinen aufrichtigen Dank dafür aussprechen, dass sie mit ihrem Engagement in diesem Jahr die Tätigkeit der FMH für die Mitglieder sichtbarer gemacht und zur Weiterentwicklung dieser verschiedenen Förderinstrumente beigetragen haben. Ich wünsche den Mitarbeitenden des Generalsekretariats und den Mitgliedern der FMH, ihren Nächsten sowie allen Leserinnen und Lesern der SÄZ ein frohes Weihnachtsfest und ein gutes neues Jahr.

Anne-Geneviève Bütikofer, Generalsekretärin der FMH 